INPLASY

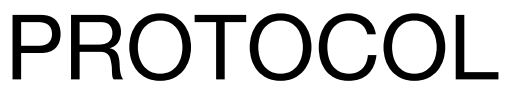

To cite: Yang et al. The improvement of the Lower Limb exoskeletons on the gait of patients with spinal cord injury: A protocol for systematic review and metaanalysis. Inplasy protocol 202180095. doi: 10.37766/inplasy2021.8.0095

Received: 25 August 2021

Published: 25 August 2021

Corresponding author: Xiali Xue

390231882@qq.com

Author Affiliation: Institute of Sports Medicine and Health, Chengdu Sport University.

\section{Support: No.}

Review Stage at time of this submission: Preliminary searches.

Conflicts of interest: None declared.

\section{The improvement of the Lower Limb exoskeletons on the gait of patients with spinal cord injury: A protocol for systematic review and meta-analysis}

Yang, $\mathrm{X}^{1} ; \mathrm{Tu}, \mathrm{H}^{2} ; \mathrm{Xue}, \mathrm{X}^{3}$.

Review question / Objective: The purpose of this systematic review and meta-analysis was to determine the efficacy of lower extremity exoskeletons in improving gait function in patients with spinal cord injury, compared with placebo or other treatments.

Condition being studied: Spinal Cord Injury (SCI) is a severely disabling disease. In the process of $\mathrm{SCI}$ rehabilitation treatment, improving patients' walking ability, improving their self-care ability, and enhancing patients' self-esteem is an important aspect of their return to society, which can also reduce the cost of patients, so the rehabilitation of lower limbs is very important. The lower extremity exoskeleton robot is a bionic robot designed according to the principles of robotics, mechanism, bionics, control theory, communication technology, and information processing technology, which can be worn on the lower extremity of the human body and complete specific tasks under the user's control. The purpose of this study was to evaluate the effect of the lower extremity exoskeleton on the improvement of gait function in patients with spinal cord injury.

INPLASY registration number: This protocol was registered with the International Platform of Registered Systematic Review and Meta-Analysis Protocols (INPLASY) on 25 August 2021 and was last updated on 25 August 2021 (registration number INPLASY202180095).

\section{INTRODUCTION}

Review question / Objective: The purpose of this systematic review and meta-analysis was to determine the efficacy of lower extremity exoskeletons in improving gait function in patients with spinal cord injury, compared with placebo or other treatments.

Condition being studied: Spinal Cord Injury (SCI) is a severely disabling disease. In the 
process of $\mathrm{SCl}$ rehabilitation treatment, improving patients' walking ability, improving their self-care ability, and enhancing patients' self-esteem is an important aspect of their return to society, which can also reduce the cost of patients, so the rehabilitation of lower limbs is very important. The lower extremity exoskeleton robot is a bionic robot designed according to the principles of robotics, mechanism, bionics, control theory, communication technology, and information processing technology, which can be worn on the lower extremity of the human body and complete specific tasks under the user's control. The purpose of this study was to evaluate the effect of the lower extremity exoskeleton on the improvement of gait function in patients with spinal cord injury.

\section{METHODS}

Participant or population: All spinal cord injury patients, regardless of age, sex, race, country, and disease.

Intervention: The experimental group wore a lower limb exoskeleton for rehabilitation training.

Comparator: The control group received a placebo or other treatment techniques.

Study designs to be included: Randomized Controlled Trials (RCTs).

Eligibility criteria: All randomized controlled trials (RCTs) reported will be included without regional and language restrictions. Animal studies, cohort studies, casecontrolled studies, case reports, and expert experience will be excluded.

Information sources: The following electronic databases will be searched from inception to June 2021: PubMed, the Cochrane Library, Web of Science, China National Knowledge Infrastructure (CNKI), WanFang Data, Weipu Electronics. In addition, reference lists of the included studies were manually searched to identify additional relevant studies.
Main outcome(s): The improvement indicators of lower limb gait function in patients with spinal cord injury mainly included Berg Balance scale, BBS; Time up and go test, TUG; 10-meter walk test, 10 MWT; Fugl-Meyer assessment for the lower-extremity, FMA-LE.

Quality assessment / Risk of bias analysis: Grading of Recommendations Assessment, Development, and Evaluation (GRADE) will be used to assess the quality of evidence, which is classified as high, moderate, low, or very low. Assessments include the risk of bias, inconsistency, indirectness, impreciseness, and other considerations. The Cochrane Collaboration tool will be used to assess the risk of bias.

Strategy of data synthesis: The metaanalysis will be performed posthoc when an adequate number of sufficiently homogeneous studies are found after data extraction, using Review Manager software. Aggregate-level data will be used for meta-analysis. Data will be pooled using the random-effects model. Continuous outcomes measured on the same scale will be synthesized using mean difference and reported as a mean difference with a $95 \%$ confidence interval (CI). The Review Manager (RevMan) V.5.4 software will be used for statistical analysis.

Subgroup analysis: The subgroup analysis will be conducted if there is substantial heterogeneity between the study results, following items will be considered: gender, age, and outcome styles.

Sensitivity analysis: We will perform sensitivity analysis based on sample size, research design, heterogeneity quality, methodological quality, and statistical model, excluding trials with low quality, and ensure the stability of analysis results.

Country(ies) involved: China.

Keywords: Spinal cord injury; SCI; Lower Limb; Exoskeletons; Gait. 
Contributions of each author:

Author 1 - Xinwei Yang - Conceptualization;

Formal analysis; Methodology; Software;

Writing - original draft.

Email: 1434997155@qq.com

Author 2 - Huan Tu - Formal analysis; Data

curation; Writing - review \& editing.

Email: 1950218527@qq.com

Author 3 - Xiali Xue - Methodology;

Software; Writing - review \& editing.

Email: 390231882@qq.com 